Introduction HPV causes $\sim 90 \%$ of anal cancers, with HPV16 predominating. Gay men, particularly HIV-positive men, are at greatly increased risk of anal cancer. We describe prevalence and predictors of anal canal detection of any HPV, high-risk (hr) HPV and HPV16, in a cohort of Australian gay men.

Methods SPANC is a 3-year community-recruited, prospective study of $\mathrm{HIV}(+)$ and $\mathrm{HIV}(-)$ gay men aged $\geq 35$ years. At 6monthly visits participants complete questionnaires, undergo high resolution anoscopy and anal swabs for cytology and HPV genotyping (Roche Linear Array).

Results By December 2014, 482 participants (median age 49.5 years; $31 \%$ HIV positive) had attended a baseline visit. The majority $(87 \%)$ had $\geq 1 \mathrm{HPV}$ genotype detected, with $\sim$ two thirds $(65 \%)$ having $\geq 1$ hrHPV detected. HPV16 was detected in $31 \%$ of participants. HIV positive participants and younger participants were more likely to have any HPV detected $(p=0.001$ and $\mathrm{p}=0.033$ respectively). The detection of hrHPV in univariate analyses was significantly associated with positive HIV status $(\mathrm{p}=0.001)$, currently smoking $(\mathrm{p}=0.014)$, younger age $(\mathrm{p}=$ $0.012)$, more lifetime male sexual partners $(\mathrm{p}=0.003)$, more lifetime receptive (RAI) and insertive (IAI) anal intercourse $(\mathrm{p}=$ 0.037 and $\mathrm{p}=0.002$ respectively) history of anal chlamydia ( $\mathrm{p}$ $=0.003$ ), more receptive anal behaviours in the last 6 months, including RAI ( $\mathrm{p}<0.001)$; rimming $(\mathrm{p}<0.001)$; fingering $(\mathrm{p}<$ $0.001)$. More frequent recent RAI and rimming and more lifetime IAI with condoms remained significant in multivariate analyses. HPV16 detection among HIV positive participants was four times more likely among men with a last CD4 cell count < 350 (OR 4.00, p = 0.008) and twice as likely among men with a nadir CD4 cell count $<200$ (OR 2.12, $\mathrm{p}=0.033$ ).

Conclusion Anal HPV was extremely common in this cohort of homosexual men. Prevalent HPV16 was related to low CD4 count in HIV positive men. Receptive anal sexual practices, including rimming and fingering were predictors of hrHPV detection.

Disclosure of interest statement AEG has received honoraria and research funding from CSL Biotherapies, honoraria and travel funding from Merck, and sits on the Australian advisory board for the Gardasil HPV vaccine. CKF has received honoraria, travel funding and research funding from CSL and Merck, sits on the Australian advisory board for the Gardasil HPV vaccine, and owns shares in CSL Biotherapies. SMG has had grant support from CSL Bio and GlaxoSmithKline, and lecture fees from Merck, GlaxoSmithKline and Sanofi Pasteur; in addition, has received funding through her institution to conduct HPV vaccine studies for MSD and GlaxoSmithKline and is a member of the Merck Global Advisory Board as well as the Merck Scientific Advisory Committee for HPV. RJH has received support from CSL Biotherapies and MSD. All other authors declare that they have no conflicts of interest.

\section{P10.14 FACTORS ASSOCIATED WITH HPV VACCINATION COVERAGE AT SCHOOL LEVEL DURING HPV VACCINE INTRODUCTION IN THAILAND}

${ }^{1} \mathrm{P}$ Pensuk*, ${ }^{1} \mathrm{~N}$ Wihantong, ${ }^{1} \mathrm{~W}$ Klinsupa, ${ }^{2} \mathrm{~L}$ Wechakul, ${ }^{2} \mathrm{~S}$ Pankhun, ${ }^{1} \mathrm{~S}$ Jiamsiri. ${ }^{1}$ Expanded Program on Immunization, Ministry of Public Health of Thailand; ${ }^{2}$ Bureau of General Communicable Disease, Ministry of Public Health of Thailand

\subsection{6/sextrans-2015-052270.442}

Introduction In 2014, HPV vaccination program was launched to fifth grade female students in Phra Nakhon Si Ayutthaya province in Thailand. The overall HPV vaccination coverage was
91\%, but sub-provincial HPV vaccination coverage varied due to multiple factors. This study aimed to explore factors associated with HPV vaccination coverage at school level during the introduction of HPV vaccine to the national immunisation program.

Methods A retrospective cohort study was conducted in Phra Nakhon Si Ayutthaya province, 613 fifth grade female students from 47 schools were surveyed to determine HPV vaccination coverage at school level. Teachers, healthcare workers (HCWs) and students were interviewed using standardised questionnaire to assess their knowledge and attitude about cervical cancer, HPV vaccine and HPV vaccination program. Furthermore, we explored programmatic activities that might affect HPV vaccination performance then conducted the multiple linear regression analysis to determine their relationship to HPV vaccination coverage at school level.

Results HPV vaccination coverage at schools range from 61.5 to 100 percent. Multiple linear regression analysis revealed that "HCWs knowledge that HPV vaccine safety and efficacy was carefully reviewed prior to registration" ( $\mathrm{B}=42.2$, $\mathrm{p}$-value < 0.01 ) and "HCWs supported HPV vaccine introduction in the national immunisation program" $(\mathrm{B}=8.9, \mathrm{p}$-value $=0.01)$ related to increasing HPV vaccination coverage, while "providing vaccine information statement (VIS) to parent" shown a negative relationship $(B=-7.6, p$-value $=0.04)$. However, there was no evidence that teacher or student knowledge and attitude were related to HPV vaccination coverage at school level.

Conclusion HCWs' confidence in HPV vaccine efficacy and safety and their support to HPV vaccine as a part of national immunisation program related to better HPV vaccine coverage, however, providing VIS to parents prior to HPV vaccination shown negative impact on HPV vaccination coverage at school level during HPV vaccine introduction.

Disclosure of interest statement This study was supported by the Department of Disease Control, Ministry of Public Health of Thailand. No pharmaceutical grant was received in the development of this study.

\section{P10.15 THE EPIDEMIOLOGY OF HEPATITIS C VIRUS IN AFGHANISTAN: A SYSTEMATIC REVIEW AND META- ANALYSIS}

${ }^{1} \mathrm{H}$ Chemaitelly*, 'S Mahmud, 1,2LA Abu-Raddad. 'Infectious Disease Epidemiology Group, Weill Cornell Medical College - Qatar, Cornell University, Qatar Foundation - Education City, Doha, Qatar; ${ }^{2}$ Department of Healthcare Policy \& Research, Weill Cornell Medical College, Cornell University, New York, USA

\subsection{6/sextrans-2015-052270.443}

Background Our study aims to characterise hepatitis C virus (HCV) epidemiology in Afghanistan, as part of the Middle East and North Africa (MENA) HCV Epidemiology Synthesis Project. The MENA HCV Epidemiology Synthesis Project is an ongoing effort to characterise HCV epidemiology and inform research, policy, and programming priorities in MENA countries.

Methods We systematically reviewed and synthesised HCV prevalence and incidence data following PRISMA guidelines. Metaanalyses using DerSimonian-Laird random effects model with inverse weighting were implemented to estimate HCV prevalence among populations at various risk of $\mathrm{HCV}$ infection. A risk of bias assessment was incorporated.

Results Our search identified $48 \mathrm{HCV}$ prevalence and one HCV incidence measures. Our meta-analyses estimated HCV prevalence at $0.7 \%$ among the general population (range: $0.0-4.0 \%$; 95\% CI: $0.3-1.3 \%), 31.8 \%$ among people who inject drugs 